\title{
Regulation of inflammatory responses by neuregulin-1 in brain ischemia and microglial cells in vitro involves the NF-kappa B pathway
}

Lauren J. Simmons ${ }^{1}$, Monique C. Surles-Zeigler ${ }^{1}$, Yonggang Li ${ }^{2}$, Gregory D. Ford ${ }^{3}$, Gale D. Newman ${ }^{1}$ and Byron D. Ford ${ }^{2 *}$

\begin{abstract}
Background: We previously demonstrated that neuregulin-1 (NRG-1) was neuroprotective in rats following ischemic stroke. Neuroprotection by NRG-1 was associated with the suppression of pro-inflammatory gene expression in brain tissues. Over-activation of brain microglia can induce pro-inflammatory gene expression by activation of transcriptional regulators following stroke. Here, we examined how NRG-1 transcriptionally regulates inflammatory gene expression by computational bioinformatics and in vitro using microglial cells.

Methods: To identify transcriptional regulators involved in ischemia-induced inflammatory gene expression, rats were sacrificed $24 \mathrm{~h}$ after middle cerebral artery occlusion (MCAO) and NRG-1 treatment. Gene expression profiles of brain tissues following ischemia and NRG-1 treatment were examined by microarray technology. The Conserved Transcription Factor-Binding Site Finder (CONFAC) bioinformatics software package was used to predict transcription factors associated with inflammatory genes induced following stroke and suppressed by NRG-1 treatment. NF-kappa B (NF-kB) was identified as a potential transcriptional regulator of NRG-1-suppressed genes following ischemia. The involvement of specific NF-kB subunits in NRG-1-mediated inflammatory responses was examined using N9 microglial cells pre-treated with NRG-1 (100 ng/ml) followed by lipopolysaccharide (LPS; $10 \mu \mathrm{g} /$ $\mathrm{ml}$ ) stimulation. The effects of NRG-1 on cytokine production were investigated using Luminex technology. The levels of the p65, p52, and RelB subunits of NF-kB and IkB-a were determined by western blot analysis and ELISA. Phosphorylation of IkB-a was investigated by ELISA.

Results: CONFAC identified 12 statistically over-represented transcription factor-binding sites (TFBS) in our dataset, including NF-kBP65. Using N9 microglial cells, we observed that NRG-1 significantly inhibited LPS-induced TNFa and IL-6 release. LPS increased the phosphorylation and degradation of IkB-a which was blocked by NRG-1. NRG-1 also prevented the nuclear translocation of the NF-kB p65 subunit following LPS administration. However, NRG-1 increased production of the neuroprotective cytokine granulocyte colony-stimulating factor (G-CSF) and the nuclear translocation of the NF-kB p52 subunit, which is associated with the induction of anti-apoptotic and suppression of pro-inflammatory gene expression.

(Continued on next page)
\end{abstract}

\footnotetext{
* Correspondence: byron.ford@medsch.ucr.edu

${ }^{2}$ Division of Biomedical Sciences, University of California-Riverside School of

Medicine, 900 University Ave, Riverside, CA 92521, USA

Full list of author information is available at the end of the article
} 
(Continued from previous page)

Conclusions: Neuroprotective and anti-inflammatory effects of NRG-1 are associated with the differential regulation of NF-kB signaling pathways in microglia. Taken together, these findings suggest that NRG-1 may be a potential therapeutic treatment for treating stroke and other neuroinflammatory disorders.

Keywords: Bioinformatics, Gene expression, Inflammation, Ischemia, Microarray, Neuregulin, Stroke, Transcription factor-binding site (TFBS)

Abbreviations: BSA, Bovine serum albumin; CONFAC, Conserved Transcription Factor-Binding Site Finder; DMEM, Dulbecco's modified Eagle's media; ECA, External carotid artery; ELISA, Enzyme-linked immunosorbent assay; FBS, Fetal bovine serum; ICA, Internal carotid artery; IL-13, Interleukin-13; IkB, Inhibitory kappa B; IKK, IkB kinase; IPA, Ingenuity Pathway Analysis; LPS, Lipopolysaccharide; MCA, Middle cerebral artery; MCAO, Middle cerebral artery occlusion; NF-kB, Nuclear factor kappa B; NIK, NF-kB-inducing kinase; NO, Nitric oxide; NRG-1, Neuregulin-1; PBS, Phosphate-buffered saline; PVDF, Polyvinylidene difluoride membranes; ROS, Reactive oxygen species; SDS-PAGE, Sodium dodecyl sulfate-polyacrylamide gel electrophoresis; TFBS, Transcription factor-binding sites; TNF-a, Tumor necrosis factor $a$; TTC, Triphenyltetrazolium chloride

\section{Background}

The actions of neuregulins have been demonstrated in normal brain function and in neuroprotection following cerebral ischemia. Studies from our laboratory and others demonstrated that neuregulin-1 (NRG-1) reduced ischemia-induced neuronal death in rodent models of focal stroke [1-3]. NRG-1 was neuroprotective with a therapeutic window of $>13 \mathrm{~h}$ and significantly improved neurological function [2, 4]. Neuroprotection by NRG-1 was also associated with the inhibition of pro-inflammatory gene expression following ischemia $[2,3,5-8]$. The anti-inflammatory effects of NRG-1 have also been shown in other animal models of neuroinflammation including cerebral malaria and nerve agent intoxication $[9,10]$ and in vivo using cultured macrophages and microglia $[3,11]$. However, the cellular and molecular mechanisms involved in the immunomodulatory effects of NRG-1 remain unclear.

Neuronal death following ischemic stroke involves the induction of genes associated with a number of cellular functions, including apoptosis, inflammation, and oxidative stress $[12,13]$. The initial inflammatory response following brain injury is mediated by the activation of its resident inflammatory cells, the microglia [14-16]. Activated microglia release inflammatory mediators such as reactive oxygen species (ROS), nitric oxide (NO), tumor necrosis factor $\alpha$ (TNF- $\alpha$ ), and interleukin-1 $\beta$ (IL13) [17-19]. While the initial activation of microglia is the brain's attempt to protect neurons, the over-activation of microglia leads to uncontrolled inflammation which exacerbates neuronal death [18, 20, 21]. Nuclear factor kappa $\mathrm{B}(\mathrm{NF}-\mathrm{kB})$ is a transcription factor widely known to be associated with inflammatory responses following ischemia and other neuroinflammatory disorders [22-24]. There are five members of the NF- $\mathrm{kB}$ family which include $\mathrm{p} 65$ (RelA), RelB, c-Rel, p50/105 (NF-kB1), and p52/p100 (NF$\mathrm{kB} 2$ ), which exist in unstimulated cells as homo or heterodimers bound to inhibitory $\mathrm{kB}$ (IkB) family proteins. Activation of the canonical NF-kB pathway triggers IkB kinase (IKK) activity, leading to phosphorylation and degradation of $\mathrm{IkB}$ proteins and the release of NF-kB p65/p50 heterodimers. The released p65/p50 NF-kB dimers translocate from the cytoplasm to the nucleus where they bind to specific DNA sequences and promote transcription of target genes $[23,24]$. In the canonical pathway, NF-kB activation triggers the production of pro-inflammatory cytokines and NO $[18,25,26]$. Studies have shown that inhibition of the canonical NF-kB pathway prevents the production of pro-inflammatory cytokines [27-29]. The activation of the NF-kB alternative/non-canonical pathway involves NF-kB-inducing kinase (NIK), a member of the MAP kinase family, which activates IKK $\alpha$, resulting in the translocation of RelB/p52 NF-kB heterodimers to the nucleus [30-34]. The alternative NF-kB pathway has been shown to stimulate the production of anti-apoptotic and anti-inflammatory molecules [31, 35-39]. The effects of NRG-1 have been shown to be mediated by NF-kB signaling during tumorigenesis in cancers and axonal myelination [40-45]. Specifically, NRG-1 has been shown to activate NIK through its association with the NRG-1's erbB receptors [46].

To identify transcriptional regulators involved in ischemia-induced inflammatory gene expression, we examined gene expression profiles of brain tissues following ischemia and NRG-1 treatment. Using Conserved Transcription Factor-Binding Site Finder (CONFAC) software, we performed computational analysis to predict transcriptional regulators of genes that were induced following ischemic stroke but were downregulated by NRG-1. CONFAC identifies conserved transcription factor-binding sites significantly over-represented in promoter regions of a set of genes of interest compared to random control set of genes [47]. NF-kB was identified as one of the top potential regulators of NRG-1-suppressed 
genes following ischemia. Using N9 microglial cells, we observed that NRG-1 blocked the phosphorylation and degradation of $\mathrm{IkB}-\alpha$, leading to the attenuation of classical NF-kB activation. NRG-1 increased the nuclear translocation of the p52 subunit of NF-kB and the levels of anti-apoptotic cytokines G-CSF and IL-9, suggesting a role for the alternative NF-kB pathway. Understanding these mechanisms will provide insight into the molecular processes behind the neuroprotective and anti-inflammatory abilities of NRG-1.

\section{Methods}

\section{Transient middle cerebral artery occlusion (MCAO)}

All animals were treated humanely and with regard for alleviation of suffering and pain. All surgical protocols involving animals were performed by sterile/aseptic techniques and were approved by the Institutional Animal Care and Use Committee at Morehouse School of Medicine prior to the initiation of experimentation. Adult male Sprague-Dawley rats (250-300 g; Charles River Laboratory International, Inc., USA) were housed in standard cages in a temperature-controlled room $\left(22 \pm 2{ }^{\circ} \mathrm{C}\right)$ on a 12-h reverse light-dark cycle. Food and water were provided ad libitum.

Animals were randomly allocated into three groups: sham (control), middle cerebral artery occlusion $(\mathrm{MCAO})+$ vehicle treatment $(\mathrm{MCAO})$ and $\mathrm{MCAO}+$ NRG-1 (MCAO + NRG1). Rats were anesthetized with a ketamine/xylazine solution $(100 / 10 \mathrm{mg} / \mathrm{kg}$, i.p.) prior to surgery. After anesthesia administration, a rectal probe monitored the core body temperature and a Homoeothermic Blanket Control Unit (Harvard Apparatus, Hollister, MA) was used to ensure the body temperature maintained at $37{ }^{\circ} \mathrm{C}$. Cerebral blood flow was monitored throughout the length of the surgery by a continuous laser Doppler flowmeter (Perimed, Ardmore, PA), with a laser Doppler probe placed $7 \mathrm{~mm}$ lateral and $2 \mathrm{~mm}$ posterior to bregma in a thinned cranial skull window.

Rats in the treatment groups (MCAO and $\mathrm{MCAO}+$ NRG1) were subjected to a left transient MCAO. MCAO was induced by the intraluminal suture method as previously described [3]. Briefly, a 4-cm length 4-0 surgical monofilament nylon suture coated with silicon (Doccol Corp., Sharon, MA) was inserted from the external carotid artery (ECA) into the internal carotid artery (ICA) and then into the Circle of Willis, to occlude the origin of the left middle cerebral artery (MCA). After $1.5 \mathrm{~h}$ of ischemia, the nylon suture was removed and the ischemic brain was reperfused for $22.5 \mathrm{~h}$ before sacrifice. Rats in the sham control group underwent the same procedure as those in the treatment groups, but a filament was not inserted into the ICA. Animals were randomly assigned into treatment groups and either administered $50 \mu \mathrm{l}$ of NRG-1 $\beta$ reconstituted with $1 \%$ bovine serum albumin (BSA) in phosphate-buffered saline (PBS) (MCAO + NRG1; $20 \mu \mathrm{g} / \mathrm{kg}$; EGF-like domain, R\&D Systems, Minneapolis, MN) or vehicle (MCAO; $1 \%$ BSA in PBS) All treatments were administered by bolus injection into the ICA through ECA immediately before MCAO. Animals were sacrificed $24 \mathrm{~h}$ after MCAO. All NRG-1 and vehicle treatment studies were performed in a blinded manner.

\section{RNA preparation and microarray analysis}

Microarray analysis was performed as we previously described [3, 48, 49]. Animals were sacrificed, and their brains removed $24 \mathrm{~h}$ following MCAO. Removed brains were sliced into $2-\mathrm{mm}$ coronal sections (approximately +3.0 to -5.0 from bregma) using a brain matrix. Tissue from the ipsilateral tissue from the two middle slices ( +1 to -3 from bregma) of MCAO treated $(n=3)$ and MCAO + NRG1 treated $(n=3)$ and sham animals $(n=3)$ were used for RNA isolation, while the two outer slices of tissue were used for staining with 2,3,5-triphenyltetrazolium chloride (TTC), in order to confirm infarct formation. Total RNA extraction was performed using TRIzol Reagent (Life Technologies, Rockville, MD, USA), cleaned (RNAqueous Kit Ambion, Austin, TX, USA), and converted to double-stranded complementary DNA (cDNA) (Invitrogen, Superscript Choice System, Carlsbad, CA, USA) using T7-(dT)24 primer. Cleanup of doublestranded cDNA used Phase Lock Gels (Eppendorf, Westbury, NY, USA)-Phenol/Chloroform/Isoamyl Alcohol (Sigma, St. Louis, MO, USA). cRNA was synthesized using a RNA transcript labeling kit (Enzo Diagnostics, Farmingdale, NY, USA). Biotin-labeled cRNA was cleaned up using a GeneChip Sample Cleanup Module (Affymetrix Inc., Santa Clara, CA, USA) and quantified using a spectrophotometer. Twenty micrograms of the in vitro transcription product was fragmented by placing at $94{ }^{\circ} \mathrm{C}$ for $35 \mathrm{~min}$ in fragmentation buffer. Following fragmentation, $15 \mu \mathrm{g}$ of the biotinylated cRNA was hybridized to an Affymetrix Rat Genome U34A GeneChip. The chips were hybridized at $45{ }^{\circ} \mathrm{C}$ for $16 \mathrm{~h}$, and then washed, stained with streptavidin-phycoerythrin, and scanned according to the manufacturing guidelines.

\section{Microarray data analysis}

Affymetrix Expression Console software (Affymetrix, Santa Clara, CA) was used for initial data processing. Affymetrix microarrays contain the hybridization, labeling, and housekeeping controls that help determine the success of the hybridizations. The Affymetrix Expression Analysis algorithm uses the Tukey's biweight estimator to provide a robust mean signal value and the Wilcoxon's rank test to calculate a significance or $p$ value and detection call for each probe set. The detection $p$ value is 
calculated using a discrimination score (R) for all probes. The discrimination score is a basic property of a probe pair that describes its ability to detect its intended target. It measures the target-specific intensity differences of the probe pair (perfect match $(\mathrm{PM})$ - mismatch $(\mathrm{MM})$ ) relative to its overall hybridization intensity $(\mathrm{PM}+\mathrm{MM})$. Background estimation is provided by a weighted average of the lowest $2 \%$ of the feature intensities. Mismatch probes are utilized to adjust the PM intensity. Linear scaling of the feature level intensity values, using the trimmed mean, is the default to make the means equal for all arrays being analyzed. False-negative and false-positive rates are minimized by subtracting nonspecific signal from the PM probe intensities and performing an intensity-dependent normalization at the probe set level. Calculation of fold change and the presence of genes in the tissue were analyzed in Microsoft Excel. Changes in gene expression were compared between the control (sham) and following MCAO and MCAO + NRG-1 treatment. Gene expression values that increased or decreased by twofold or more were statistically significant $(p<0.05)$ using two-way ANOVA. The list of genes upregulated twofold or more by ischemia and decreased $50 \%$ or more by NRG-1 was analyzed using Ingenuity Pathway Analysis (IPA) software (Qiagen, Redwood City, CA; www.ingenuity.com) overlaid onto a global molecular network developed from information contained in the Ingenuity Knowledge Base. Fischer' $\mathrm{s}$ exact test was used to calculate a $p$ value determining the probability that each biological function and/or disease assigned to that network is due to chance alone. The functions, canonical pathways, and gene networks that were most significant to the dataset were identified. IPA identified 64 inflammatory genes that were upregulated twofold or more by $\mathrm{MCAO}$ and reduced $50 \%$ or more by NRG-1.

\section{CONFAC analysis}

Using CONFAC software, we further examined the IPAidentified inflammatory genes [47, 48]. A tab-delimited text file containing the gene name and RefSEQ ID of the clusters of interest (genes) was uploaded to the CONFAC web browser interface (http://confac.emory.edu/). CONFAC software identified mouse orthologs from the uploaded gene list (from UCSC and ENSEMBL genomes). CONFAC software next identified significantly conserved sequences ( $e$ value $<0.001$ ), which were analyzed for transcription factor-binding sites (TFBS) using MATCH $^{\text {тм }}$ software. The final output table consisted of the cohort list of genes (column) and the position weight matrix (collected in TRANSFAC ${ }^{\bullet}$ database) identified potential TFBS and the number of TFBS determined for each gene of interest. The CONFAC program then allowed for additional analysis using the MannWhitney $U$ test, which facilitated the statistical analysis of TFBS over-represented in the sample dataset compared to the seven control random control datasets provided by CONFAC.

\section{N9 microglial cell culture}

The murine N9 microglial cell line was kindly provided by Dr. Celine Beamer (Department of Biomedical and Pharmaceutical Sciences, University Montana). N9 cells were cultured in Dulbecco's modified Eagle's media (DMEM) with $4 \mathrm{mM}$ L-glutamine, supplemented with $10 \%$ fetal bovine serum (FBS), streptomycin $(100 \mathrm{U} / \mathrm{ml})$, and penicillin $(100 \mathrm{U} / \mathrm{ml})$. The cells were maintained in $95 \%$ air and $5 \% \mathrm{CO}_{2}$-humified atmosphere at $37{ }^{\circ} \mathrm{C}$ and were passaged every 3 days. For culturing with various stimulants, N9 cells were plated into 12-well plates in prepared culture medium and NRG-1 and lipopolysaccharide (LPS) were applied at the following final concentrations: LPS $(10 \mu \mathrm{g} / \mathrm{ml})$ and NRG-1 $(100 \mathrm{ng} / \mathrm{ml}$ in $1 \%$ BSA/PBS; recombinant human neuregulin1-beta1 EGF-like domain, R\&D Systems). N9 cells were either pretreated with NRG-1 (100 ng/ml) for $24 \mathrm{~h}$ followed by stimulation with LPS $(10 \mu \mathrm{g} / \mathrm{ml})$ or treated with LPS or NRG-1 alone for the indicated times (1-24 h).

\section{Luminex multiplex enzyme-linked immunosorbent assay (ELISA)}

Cell lysates were taken at 1, 3, 6, and 24 h time points following LPS treatment. The cytokine release was measured in cell culture supernatants by Luminex technology using the manufacturer's protocol (Bio-Rad, Hercules, CA). Data analysis was performed using Bio-Plex Manager software on the Bio-Plex 200 system. Experiments were performed four times in duplicate wells.

\section{Protein isolation and western blot}

Proteins were isolated using whole cell extraction reagent M-PER (Pierce Biotechnology, Rockford, IL) or NE-PER Nuclear and Cytoplasmic Extraction Reagent (Thermo Scientific) according to the manufacturer's instructions. Protein concentrations were determined by Bradford protein assay (Bio-Rad). Fifty micrograms of protein lysate was subjected to sodium dodecyl sulfatepolyacrylamide gel electrophoresis (SDS-PAGE) and transferred to polyvinylidene difluoride membranes (PVDF) (Bio-Rad, CA). The membranes were blocked with $3 \%$ nonfat dry milk in $0.1 \%$ Tween-20/Tris-buffered saline for $1 \mathrm{~h}$ at room temperature and subsequently incubated with the primary antibodies overnight, followed by incubation in corresponding secondary horseradish peroxidase-conjugated secondary antibody for $1 \mathrm{~h}$ at room temperature. Blots were developed using immuno-western star ECL detection kits (Bio-Rad). The optical densities of the antibody-specific bands were analyzed by a Luminescent Image Analyzer, LAS-4000 
(Fuji, Japan). The following antibodies from Santa Cruz Biotechnology (Santa Cruz, CA, USA) were used: NF-kB p65 (sc-372; 1:200), IkB- $\alpha$ (sc-371; 1:200), YY1 (sc-281; $1: 200)$, and $\beta$-actin (sc-130656; 1:200).

\section{IkB-a (total/phospho) InstantOne ELISA}

The phosphorylation of IkB- $\alpha$ was determined using an enzyme-linked immunosorbent assay (ELISA)-based assay kit according to the manufacturer's protocol (Affymetrix eBioscience, San Diego, CA). To analyze the results, we calculated the average values for each treatment group. This assay was run four times in duplicate.

\section{Active motif transAM NF-kB family ELISA}

Nuclear extracts were isolated using NE-PER Nuclear and Cytoplasmic Extraction kit according to the manufacturer's protocol (Thermo Scientific, Waltham, MA). ELISA was performed using the manufacturer's protocol. To analyze the results, the optical density was read using $450 \mathrm{~nm}$ with an optional reference wavelength of $655 \mathrm{~nm}$. This assay was run three times in duplicate.

\section{Statistical analysis}

Statistical analysis for ELISAs and western blot data was performed by ANOVA with Tukey's post hoc test for multiple comparisons where indicated. Significance was determined using a $p$ value less than 0.05 .

\section{Results}

CONFAC analysis predicts transcription factor NF-kB to be regulated following MCAO + NRG1

NRG-1 has been shown to prevent neuronal injury when administered before or after MCAO. In these studies, rats were treated with NRG-1 immediately prior to transient MCAO for maximal effect. In representative TTCstained coronal brain sections, rats were administered vehicle (Fig. 1a) or NRG-1 before MCAO (Fig. 1b). The white area indicates damaged neuronal cells (arrows) and red staining indicates normally functioning cells. RNA was isolated from the ipsilateral hemisphere of each experimental group (sham controls, MCAO, and MCAO + NRG1) and used to examine gene expression profiles in brain tissues from each condition. Using microarray and IPA analysis, we found that 64 inflammatory genes were increased twofold or more $24 \mathrm{~h}$ following MCAO and reduced by $50 \%$ with NRG-1 treatment (Table 1). The list included a number of well-characterized pro-inflammatory molecules, including IL-1 $\beta$, PTGS2/COX2 (protaglandin-endoperoxide synthase-2/cyclooxygenase-2), CD36, SPP1 (secreted phosphoprotein 1/osteopontin), and S100 calciumbinding proteins. IL- $1 \beta$ and PTGS2/COX2 induction by $\mathrm{MCAO}$ and in a monocytic cell line, respectively, and attenuation by NRG-1 were previously validated by qPCR [3]. CONFAC analysis software recognized 54 genes in our dataset and analyzed TFBS in the promoters of those genes. CONFAC identified 12 TFBS as over-represented in our dataset compared to the
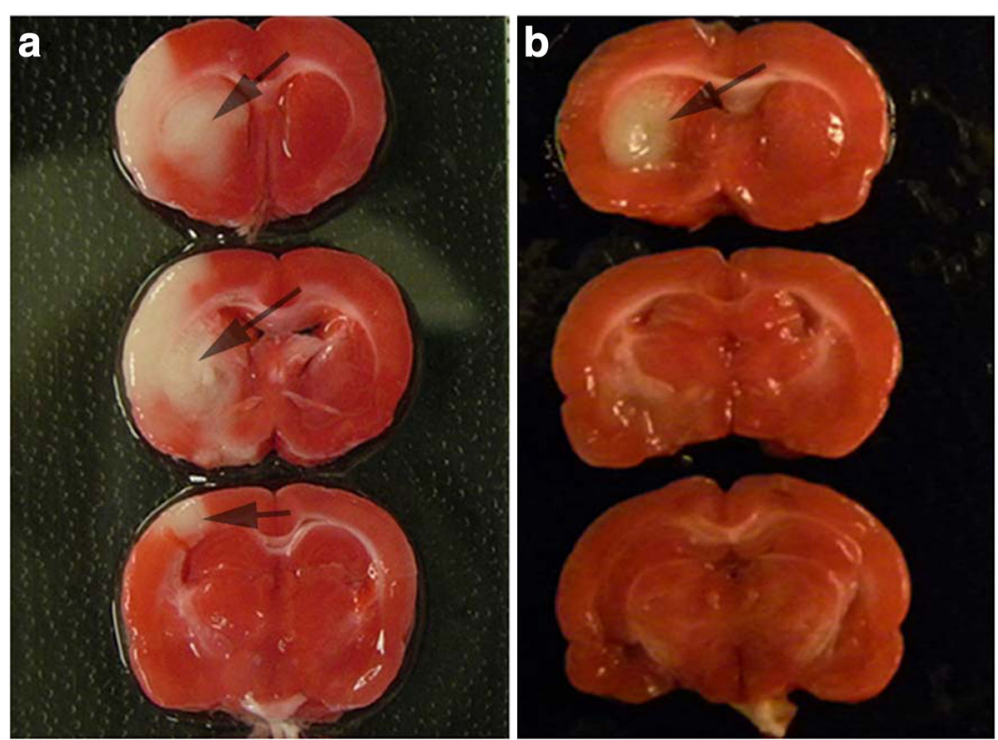

Fig. 1 Neuregulin-1 administration reduces MCAO/reperfusion-induced brain infarction. Representative TTC-stained coronal brain sections are shown. Rats were administered vehicle (a) or NRG-1 before MCAO (b). The white area indicates damaged neuronal cells (arrows) and red staining indicates normally functioning cells 
Table 1 Inflammatory genes induced by ischemia and downregulated by NRG-1

\begin{tabular}{|c|c|}
\hline A kinase anchor protein 12 & Akap12 \\
\hline Actin-related protein 2/3 complex subunit 1B & Arpclb \\
\hline Activating transcription factor 3 & Atf3 \\
\hline Annexin A1 & Anxa1 \\
\hline Annexin A2 & Anxa2 \\
\hline Benzodiazepine receptor (peripheral)-associated protein 1 & bzrp \\
\hline Brain-derived factor & Bdnf \\
\hline CAMP responsive element modulator & Crem \\
\hline Cartilage oligomeric matrix protein & Comp \\
\hline Catechol-O-methyltransferase & Comt \\
\hline CCAAT/enhancer-binding protein, delta & Cebpd \\
\hline CD36 antigen & $\operatorname{cd} 36$ \\
\hline Cellular retinoic-binding protein 2 & Crabp2 \\
\hline Crystallin, alpha B & Cryab \\
\hline Cytochrome P450 family 1 & Cyp1b1 \\
\hline Deiodinase iodothyronine type III & Dio3 \\
\hline Dyskeratosis congenita 1 & Dkc1 \\
\hline Early growth response 1 & Egr1 \\
\hline Endothelin converting enzyme 1 & Ece1 \\
\hline Fx receptor, IgG, low-affinity III & Fcgr3 \\
\hline Galanin & Gal \\
\hline Glycosylation-dependent cell adhesion molecule 1 & Glycam1 \\
\hline Guanine nucleotide-binding protein gamma 11 & Gng11 \\
\hline Guanylate-binding protein & Gbp2 \\
\hline Heat shock protein 4 & Hspa4 \\
\hline High mobility group of box 2 & Hmgb2 \\
\hline Homer scaffolding protein 1 & Homer1 \\
\hline Inhibitor of DNA binding 1 & Id1 \\
\hline Insulin-like growth factor-binding protein 3 & lgfbp3 \\
\hline Interleukin 1 beta & $\mathrm{IL} 1 \mathrm{~b}$ \\
\hline Isopentenyl-diphosphate delta isomerase 1 & Idi1 \\
\hline Kininogen 1 & Kng \\
\hline Kruppel-like factor 4 & Klf4 \\
\hline Lectin galatoside-binding soluble 2 & LgalS2 \\
\hline Lipocalin 2 & Len \\
\hline Lipopolysaccharide-binding protein & Lbp \\
\hline Lysozyme & Lyz \\
\hline Matrix gla protein & Mgp \\
\hline Matrix metallopeptidase 9 & MMP9 \\
\hline Mitogen-activated protein kinase e, E3 & Map \\
\hline Oxidized low-density lipoprotein receptor 1 & Olr1 \\
\hline Phospholipase A1 & Pspla1 \\
\hline Phosphoribosyl pyrophosphate synthase-associated protein 1 & Prpsap1 \\
\hline Phosphorylase glycogen liver & Pygl \\
\hline
\end{tabular}

Table 1 Inflammatory genes induced by ischemia and downregulated by NRG-1 (Continued)

\begin{tabular}{ll}
\hline Plasminogen activator tissue & Plat \\
Potassium channel member 12 & Kcnj12 \\
Potassium channel, two pore domain subfamily K member 3 & Kcnk3 \\
Protaglandin-endoperoxide synthase 2 & Ptgs2 \\
Rentinol-binding protein 1 & Rbp1 \\
Ret proto-oncogene & Ret \\
Ribosomal protein S15 & Rps15 \\
S100 calcium-binding protein A10 & S100a10 \\
S100 calcium-binding protein A4 & S100a4 \\
S100 calcium-binding protein A8 & S100a8 \\
S100 calcium-binding protein A9 & S100a9 \\
Secreted phosphoprotein 1 & Spp1 \\
Serpin peptidase inhibitor & Serpin1 \\
Syndecan 1 & Sdc1 \\
Thyrotropin releasing hormone & Trh \\
TIMP metallopeptidase inhibitor 1 & Timp1 \\
Transgelin & Tagln \\
V-ETS avian erythroblastosis virus E26 oncogene homolog 1 & ETS1 \\
VGF nerve growth factor inducible & Vgf \\
Vimentin & Vim \\
\hline
\end{tabular}

random control datasets. The TFBS included MAZ, NFKAPPAB65, XBP1, XFD1, SOX, ATF3, NFE2, IRF7, GABP, EBF, and CACCBINDINGFACTOR (Fig. 2). NFKAPPAB65 (NF-kB p65 subunit) has been previously associated with stroke and the effects of NRG-1 in breast cancer and neuronal myelination [40-43, 45], so we further investigated the role of NF-kB in NRG-1mediated neuroinflammation.

\section{NRG-1 suppressed the release of cytokines TNF- $a$ and IL-6 in LPS-stimulated N9 microglial cells}

We previously demonstrated the induction of IL-1 $\beta$ and monocyte chemoattractant protein 1 (MCP-1/CCL2) following MCAO and their inhibition by NRG-1 [3]. To determine whether NRG-1 inhibits the general production and release of pro-inflammatory cytokines, N9 microglia were pre-treated with NRG-1 followed by stimulation with LPS. Cytokine levels in the conditioned medium were measured by Luminex multiplex ELISA. Following LPS stimulation, there was an increase in TNF$\alpha$ production and release at 3,6 , and $24 \mathrm{~h}$ from microglial cells. NRG-1 pre-treatment significantly reduced the levels of TNF- $\alpha$ at $3 \mathrm{~h}(p=0.0003), 6 \mathrm{~h}(p=0.0011)$, and $24 \mathrm{~h}$ $(p=0.01)$ post-stimulation with LPS (Fig. 3a). LPS stimulation also increased IL-6, which was significantly reduced by NRG-1 at $6 \mathrm{~h}(p=0.02)$ post-LPS stimulation (Fig. $3 \mathrm{~b})$. 


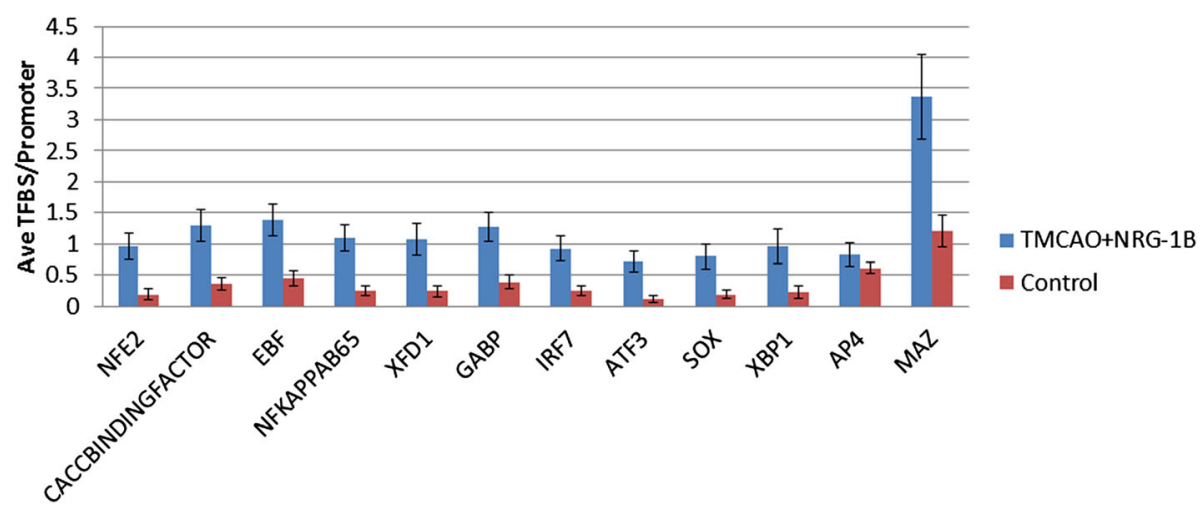

Fig. 2 Predicted transcription factor-binding site (TFBS) activity for gene promoters using CONFAC analysis. CONFAC compared our gene list to seven random control datasets to identify statistically over-represented TFBS in genes altered by stroke and reversed by NRG-1. CONFAC identified 12 TFBS that were statistically over-represented. Blue bars represent the average number of TFBS/promoter for each transcription factor in our data set. Red bars are the average number of TFBS/promoter for each transcription factor in the control datasets. $p<0.05$ for all transcription factors in the graph

\section{NRG-1 attenuated LPS-stimulated phosphorylation and degradation of IkB-a in N9 microglial cells}

In the canonical NF-kB pathway, IkB kinase (IKK) phosphorylates $\mathrm{IkB}-\alpha$ triggering its ubiquitination and degradation. This allows the NF-kB p56 and p50 subunits to translocate to the nucleus, resulting in the production and secretion pro-inflammatory cytokines and chemokines. Here, we examined whether NRG-1 regulates LPS-stimulated IkB- $\alpha$ phosphorylation in N9 microglia cells. N9 cells were either pre-treated with NRG-1 for $24 \mathrm{~h}$ followed by stimulation with LPS or treated with LPS or NRG-1 alone for the indicated times (1-24 h). Using an ELISA system, phosphorylation of IkB- $\alpha$ in microglial cell lysates increased $1 \mathrm{~h}$ post-LPS stimulation, peaked at $3 \mathrm{~h}$, and then returned to baseline 6-24 $\mathrm{h}$ following LPS stimulation (Fig. 4a, b). Pre-treatment with NRG-1 significantly reduced the phosphorylation of $\mathrm{IkB}-\alpha$ in response to LPS stimulation at both the 1- and 3-h time points. We used western blot analyses to determine the effect of NRG-1 on the degradation of IkB- $\alpha$. N9 cells were pre-treated with NRG-1 for $24 \mathrm{~h}$ followed by stimulation with LPS or treated with LPS or NRG-1 alone for 1 and $3 \mathrm{~h}$. At the 1 -h time point, there was a reduction in IkB- $\alpha$ in LPS-treated cells when compared to controls; however, this was reversed in cells pre-treated with NRG-1 followed by LPS treatment $(p=0.008)$ (Fig. 5).

\section{NRG-1 attenuated P65 nuclear translocation in N9 microglia cells}

To determine if NRG-1 had an effect on the canonical NF-kB pathway, we used nuclear extracts for ELISA and western blot to examine the translocation of the NF-kB p65 subunit identified by CONFAC. We observed an increase in nuclear translocation of p65 in N9 cells treated with LPS alone. However, LPS-induced p65 translocation was significantly reduced in cells pre-treated with NRG1 followed by LPS using ELISA (Fig. 6a; $p=0.004$ ) and western blot (Fig. 6b, $p=0.003$ ).

\section{NRG-1 increased the nuclear translocation of the P52 subunit}

It has been previously shown that NRG-1 is able to activate NF-kB-inducing kinase (NIK) which is a mediator of the alternative NF-kB pathway [46]. Here, we investigated if NRG-1 could affect LPS-mediated nuclear translocation of the p52 and RelB NF-kB subunits. LPS stimulation resulted in a decrease in nuclear p52 at 3 and $24 \mathrm{~h}$ post-treatment (Fig. 7a). NRG-1 pre-treatment significantly increased nuclear p52 levels at 3, 6, and $24 \mathrm{~h}$ post-LPS stimulation. Neither LPS nor NRG treatment altered nuclear levels of RelB in these studies (Fig. 7b).

\section{NRG-1 increased anti-apoptotic cytokines G-CSF and IL-9}

Granulocyte colony-stimulating factor (G-CSF) is a target of NF-kB and has been shown to be neuroprotective in stroke by inducing anti-apoptotic pathways [50-53]. The NF-kB inducible cytokine IL-9 has also been reported to prevent apoptosis. LPS stimulation of microglial cells did not affect G-CSF levels in medium. However, NRG-1 increased G-CSF levels significantly at 3,6 , and $24 \mathrm{~h}$ alone or in the presence of LPS (Fig. 8a). NRG-1 significantly increased IL-9 levels at $3 \mathrm{~h}$ following LPS stimulation (Fig. 8b). IL-9 was increased by all treatment conditions 6 and $24 \mathrm{~h}$ following NRG-1 and LPS administration. 


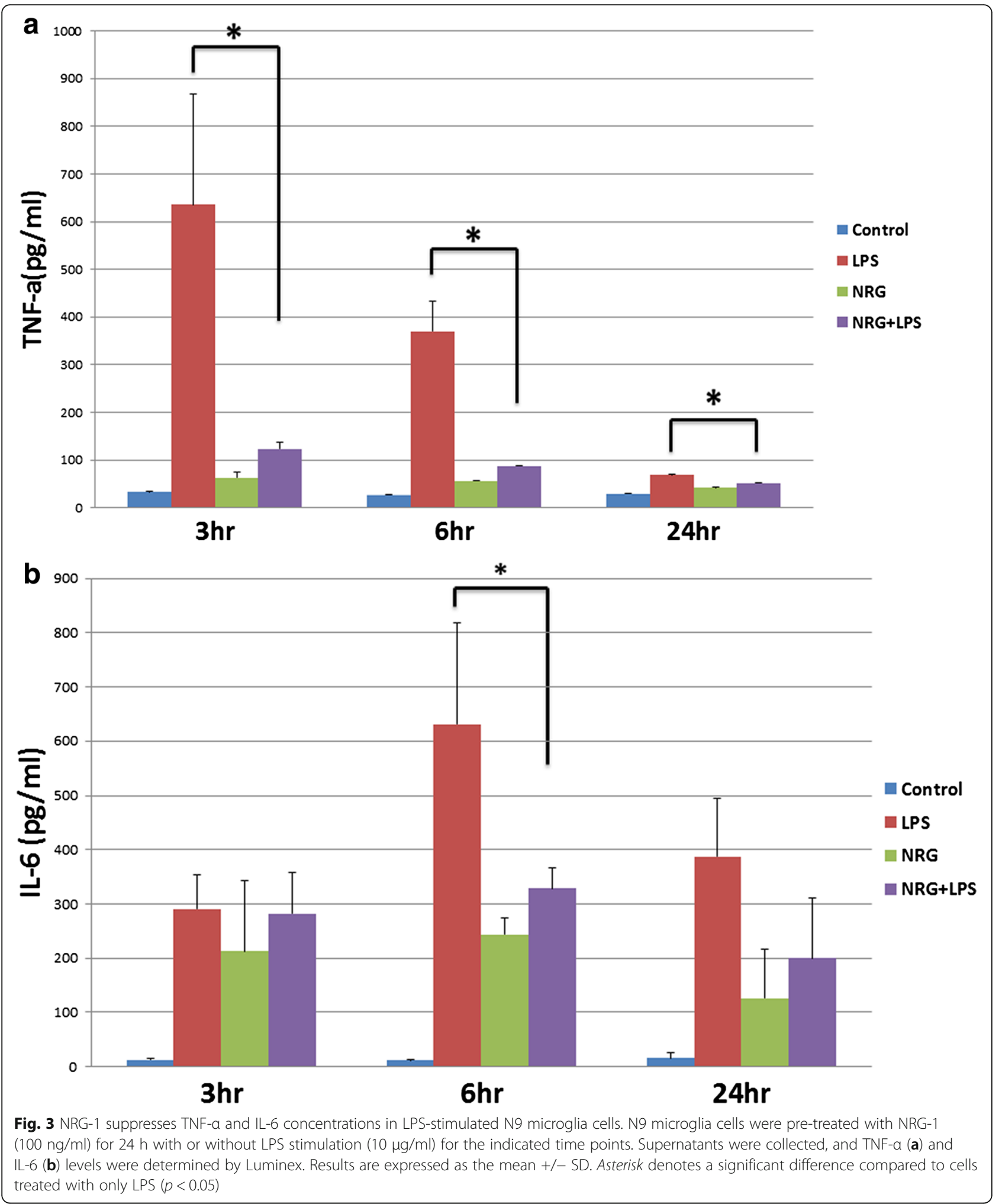

\section{Discussion}

NRG-1 has been shown to be neuroprotective and reduce inflammation in experimental models of ischemic stroke and neuroinflammation $[1-3,9,10]$; however, the molecular mechanism involved remains to be elucidated. Using CONFAC software, we were able to predict that 


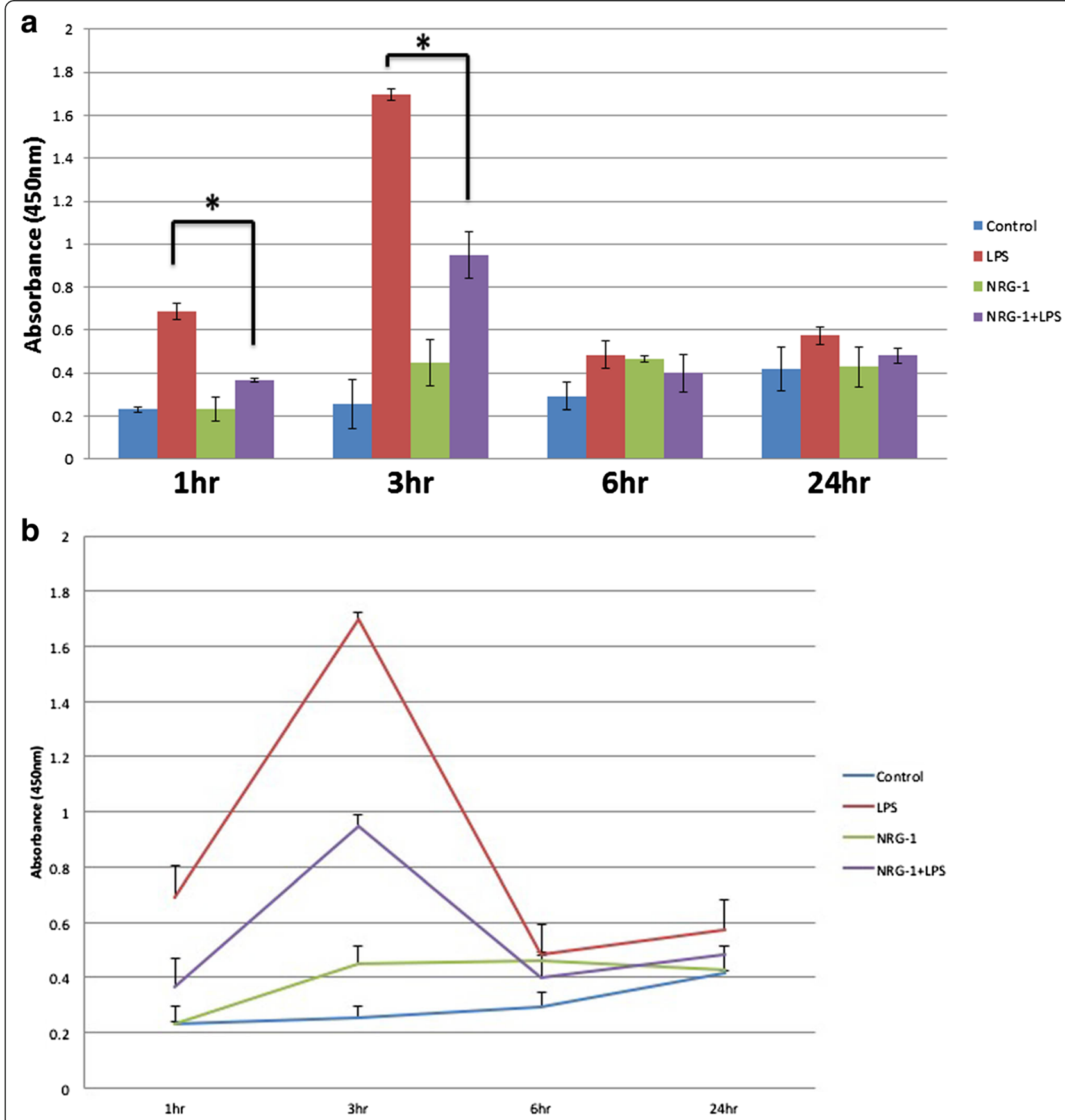

Fig. 4 NRG-1 suppresses the phosphorylation of IkB-a in LPS-stimulated N9 microglia cells. N9 microglial cells were pre-treated with $100 \mathrm{ng} / \mathrm{ml} \mathrm{NRG}-1$ for $24 \mathrm{~h}$ followed by the absence or presence of LPS $(10 \mu \mathrm{g} / \mathrm{ml})$ for indicated time points. $\mathbf{a}$, b Cell lysates were taken and were assayed using ELISA. Results were expressed as the mean $+/-$ SD. Asterisk denotes significant difference compared to cells treated with only LPS $(p<0.05)$

the ability of NRG-1 to suppress ischemia-induced proinflammatory genes involved the modulation NF-kB pathways. NF-kB is known to be a master regulator of inflammation, which is the key contributor the pathology of ischemic stroke [24]. In post-mortem human brain samples of stroke patients, the NF-kB p65 subunit in particular has been found around the necrotic infarct core [22]. The NF-kB canonical pathway involves the phosphorylation and degradation of IkB leading to the nuclear translocation of $\mathrm{p} 65 / \mathrm{p} 50 \mathrm{NF}-\mathrm{kB}$ heterodimers to the nucleus where they stimulate the production of proinflammatory molecules [23, 24]. Using microglial cell 


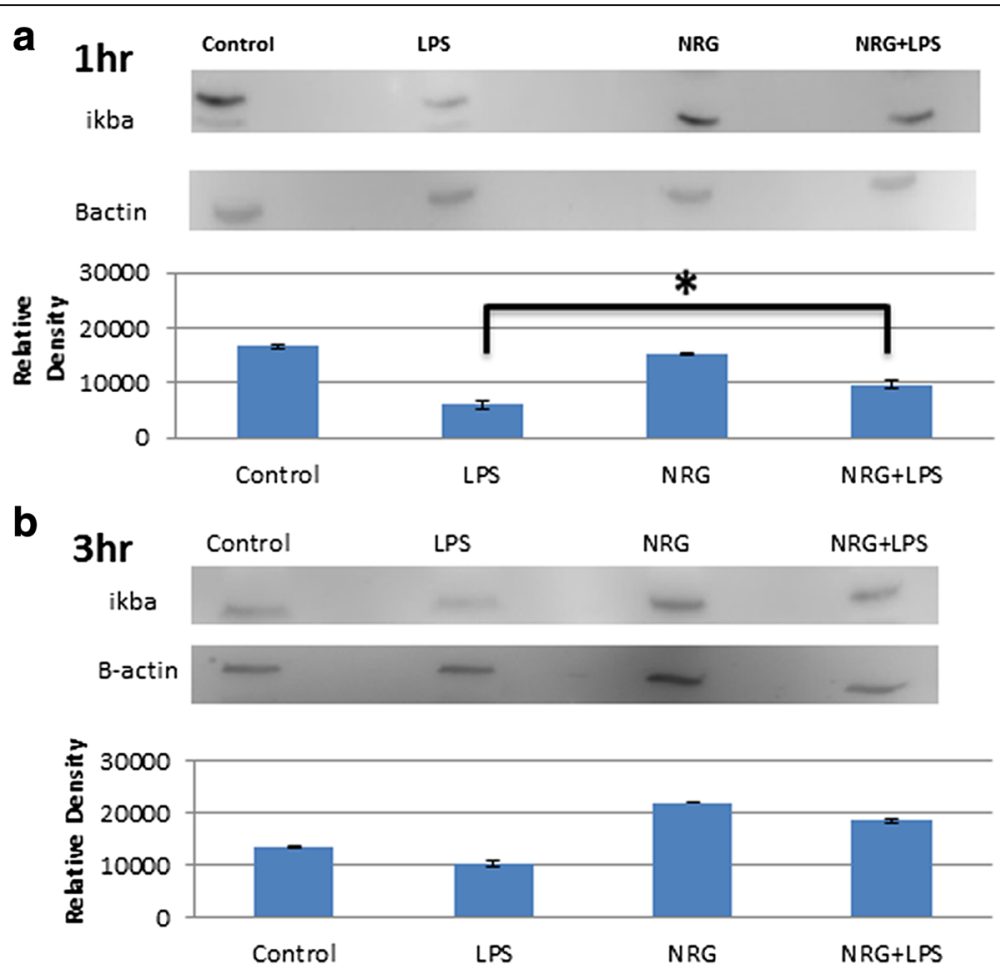

Fig. 5 NRG-1 inhibits the degradation of IkB-a in LPS-stimulated N9 microglia cells. N9 microglial cells were pre-treated with $100 \mathrm{ng} / \mathrm{ml}$ NRG-1 for $24 \mathrm{~h}$ followed by the absence or presence of LPS $(10 \mu \mathrm{g} / \mathrm{ml})$ for $1 \mathrm{~h}(\mathbf{a})$ and $3 \mathrm{~h}(\mathbf{b})$. Whole cell extracts were taken from untreated cells or cells pre-treated NRG-1 alone or the absence or presence of LPS $(10 \mu \mathrm{g} / \mathrm{ml})$ and were assayed using western blot. The band intensity was quantified using studio lite imager and is presented relative to the level of $\beta$-actin. Data are presented for three independent experiments. Results were expressed as the mean $+/-$ SD. Asterisk denotes significant difference compared to cells treated with only LPS $(p<0.05)$

cultures, we demonstrated that NRG-1 inhibited the LPS-induced phosphorylation and degradation of IkB- $\alpha$, while also reducing the nuclear translocation of p65. This suggests that the mechanisms behind the antiinflammatory/neuroprotective effects of NRG-1 involve the attenuation of the canonical NF-kB pathway.

The transcription factors identified by CONFAC did not change in mRNA expression in our analysis, including NF-kB family members. One of the primary reasons we chose a computational tool such as CONFAC is that it predicts transcription factors that change in activity, but not necessarily mRNA or protein levels. Indeed, induction of pro-inflammatory gene expression by NF-kB requires that cytoplasmic NF-kB p65/p50 translocates to the nucleus, but a change in NF-kB protein levels is not needed to mediate this effect. Over-representation of the NF-kB p65 TFBS indicates that it is more prevalent in the promoters of the genes in our dataset compared to a random set of genes. It does not indicate whether that promoter is activated or inhibited by the experimental paradigm. What our data suggest is that stroke causes transcriptional activation via the NF-kB p65-binding site, and NRG-1 prevents the binding of p65 to the TFBS by sequestering it in the cytoplasm.
The alternative NF-kB pathway is thought to have anti-inflammatory and anti-apoptotic roles [31,35]. The alternative pathway activates NIK, a member of the MAP kinase family, which activates IKK $\alpha$, resulting in the translocation of RelB/p52 NF-kB heterodimers to the nucleus. NIK-mediated activation of NF- $\mathrm{kB}$ is associated with the induction of anti-apoptotic and antiinflammatory cytokines [30, 34, 37, 38, 50, 54, 55]. The alternative pathway is delayed relative to the canonical pathway, and only certain inducers are able to induce its activation [33]. In microglial cells, NRG-1 administration increased the nuclear levels of p52. In addition to dimerization with RelB, the p52 subunit is also able to homodimerize and considered to have a repressive role due to its lack of a transcription activation domain [32]. It has been previously shown that NRG-1 activates NIK [46]. These finding indicate that NRG-1 could both activate the alternative pathway and/or suppress NF-kB transcriptional activity via p52 [23, 32, 56].

Previous studies demonstrated that NRG-1 can activate the NF-kB signaling pathway cancer cells and Schwann cells [40-46]. In these studies, it appears that NRG-1 signals by activating NF-kB p65; however, the 


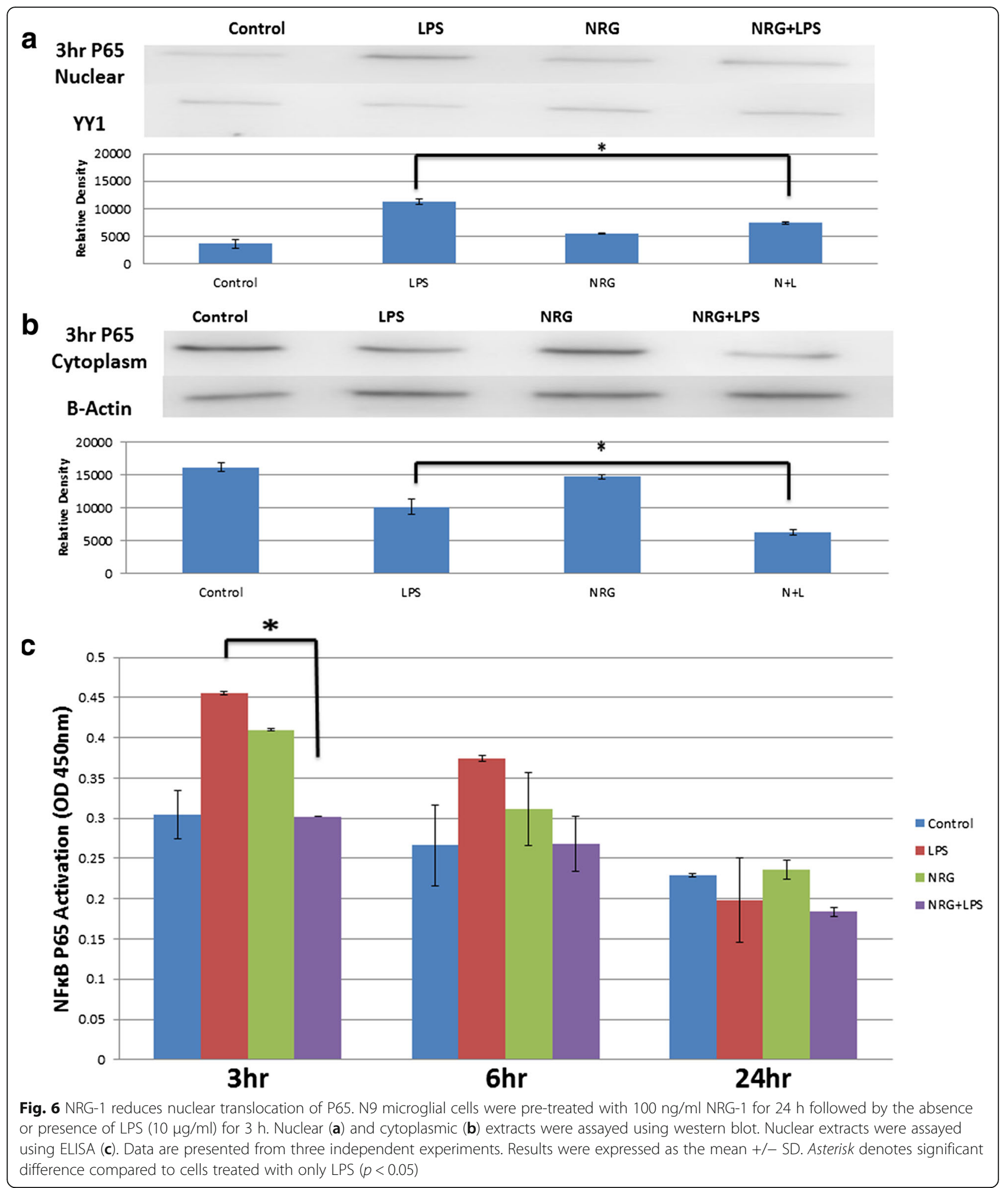

activation of NF-kB by NRG-1 in breast cancer cells is associated with the increased expression of antiapoptotic genes. The discrepancy in the findings may be due to the differential expression of erbB receptors on the cells. In cancer cells and Schwann cells, NRG-1 signals through erbB2 and erbB3 receptors. Neuroprotection in stroke by NRG-1 is mediated by erbB4 which physically associates with NIK $[46,57]$. Interestingly, 


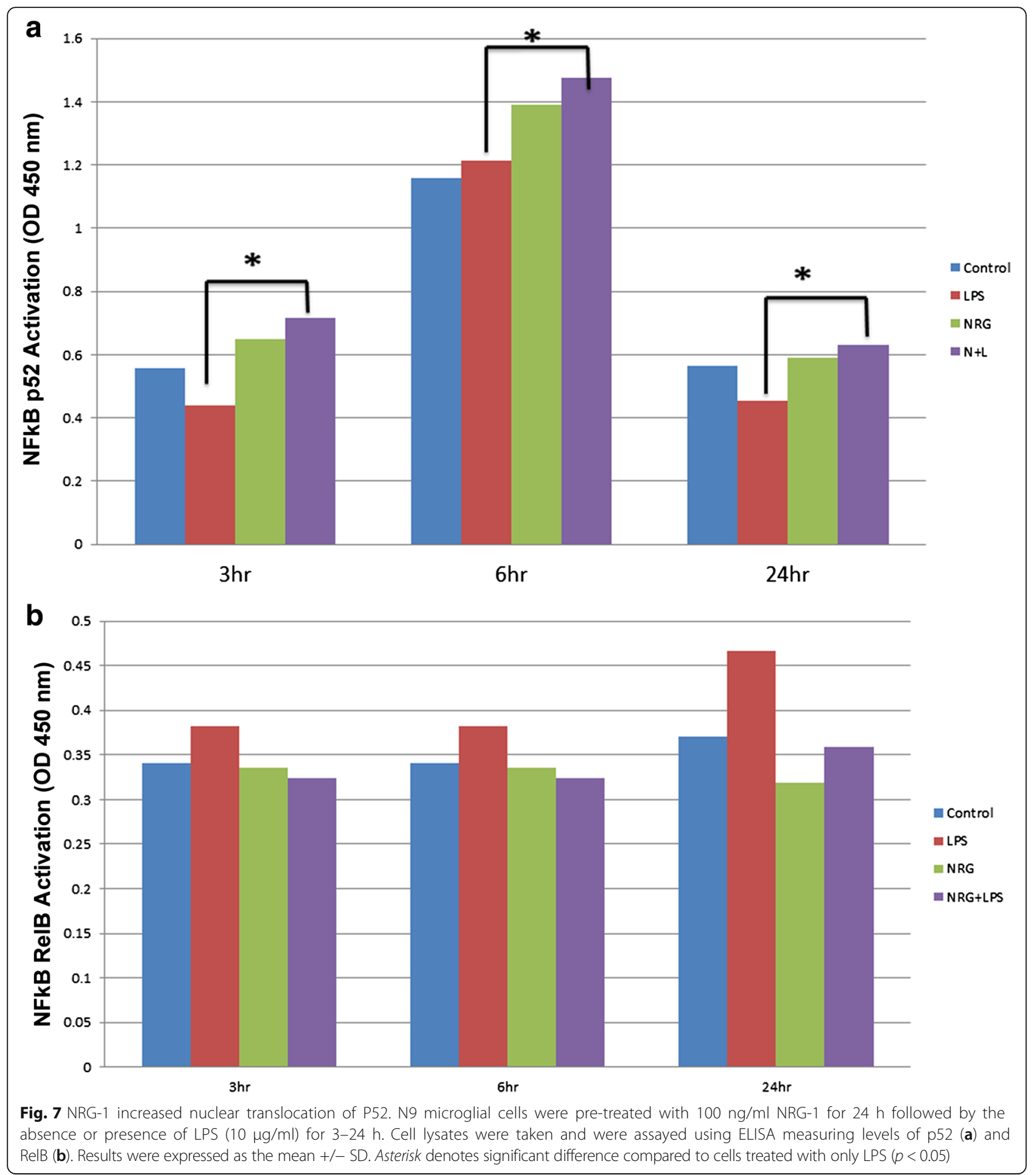

NRG-1 alone has little effect on NF-kB in our in vitro studies. So, the anti-inflammatory effects of NRG-1 appear to be contextually related to the presence on a proinflammatory response.

Consistent with regulation of the alternative NF-kB pathway, NRG-1 induced the production of G-CSF and IL-9 in cell cultures. G-CSF has been shown to be neuroprotective in ischemic stroke models by inducing anti-apoptotic pathways, and IL-9 is known to promote cell proliferation and inhibit apoptosis $[53,58]$. It has been reported that G-CSF significantly reduced the expression of microglial p65, reduced pro-inflammatory mediators, and promoted anti-inflammatory responses in models of multiple 


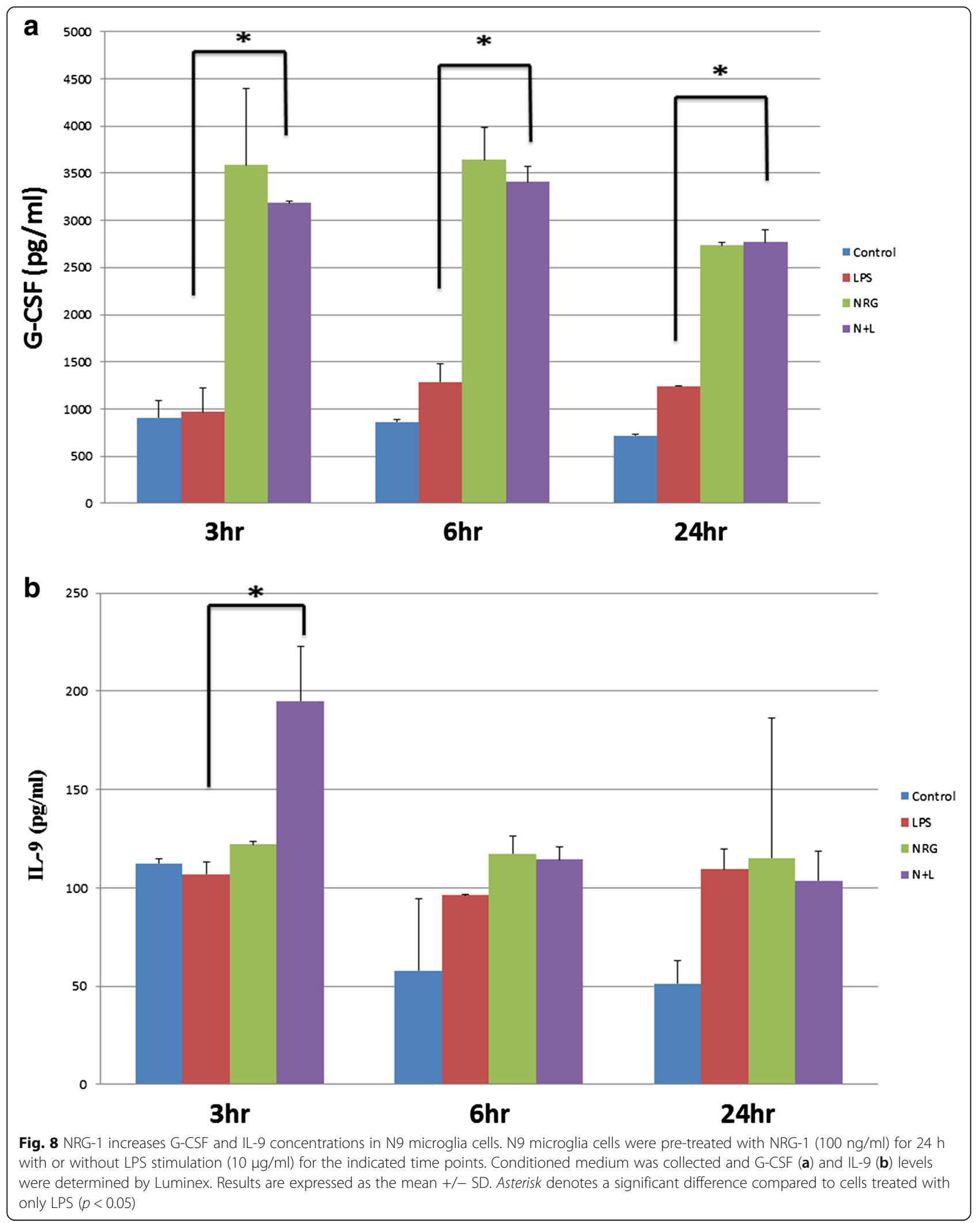


sclerosis $[59,60]$. There are reports which suggest that IL-9 may have the ability to downregulate specific sets of genes induced by NF-kB by inducing the expression of BCL-3 [61]. BCL3 is an IkB protein that specifically associates with homodimers p50 and p52 and has been shown to regulate pro-survival genes. BCL3 is also able to repress transcription by increasing $\mathrm{p} 52$ homodimer binding to $\mathrm{kB}$ sites on DNA [62].

\section{Conclusions}

Stroke is a leading cause of death and disability in the USA. There is an urgent need to better understand the pathophysiology associated with stroke in order to develop more effective therapies. Here, we show that the neuroprotective and anti-inflammatory effects of NRG-1 are associated with the regulation of both canonical and alternative NF-kB signaling pathways. NRG-1 is currently in clinical trials for heart failure and has been shown to be safe and efficacious in phase I and phase II patient studies [63, 64]. These findings have major implications for the development of NRG-1 as a clinical therapy for stroke and other inflammation-mediated disorders.

\section{Acknowledgements}

We thank Timothy Distel for technical support.

\section{Funding}

This project was supported in part by NIH grants U54 NS060659 and U54 RR026137, G12RR003034, and S21MD000101.

\section{Availability of data and materials}

Raw data are available on personal demand.

\section{Authors' contributions}

BF and LS conceived the study, participated in its design and coordination, and helped to draft the manuscript. MS, GF, and GN participated in its design and coordination of the study and helped to draft the manuscript. YL conducted the MCAO surgeries. LS and GN designed and performed the in vitro studies. LS, GF, and BF conducted the data analysis. All authors read and approved the final manuscript.

\section{Competing interests}

Patents related to the work being reported is held by the author (BF) without direct corporate involvement at the time.

\section{Consent for publication}

Not applicable

\section{Ethics approval and consent to participate}

The study protocol was approved by the Institutional Animal Care and Use Committee at Morehouse School of Medicine according to international guidelines prior to the initiation of experimentation.

\section{Author details}

${ }^{1}$ Morehouse School of Medicine, 720 Westview Drive, Atlanta 30310, Georgia. ${ }^{2}$ Division of Biomedical Sciences, University of California-Riverside School of Medicine, 900 University Ave, Riverside, CA 92521, USA. ${ }^{3}$ Georgia Highlands College, 5441 GA-20, Cartersville, GA 30121, USA.

Received: 27 June 2016 Accepted: 22 August 2016

Published online: 06 September 2016

\section{References}

1. Li Y, et al. Neuroprotection by neuregulin-1 in a rat model of permanent focal cerebral ischemia. Brain Res. 2007;1184:277-83.

2. $\mathrm{Xu} Z$, et al. Extended therapeutic window and functional recovery after intraarterial administration of neuregulin-1 after focal ischemic stroke. J Cereb Blood Flow Metab. 2006;26(4):527-35.

3. $\mathrm{Xu} Z$, et al. Neuroprotection by neuregulin-1 following focal stroke is associated with the attenuation of ischemia-induced pro-inflammatory and stress gene expression. Neurobiol Dis. 2005;19(3):461-70.

4. laci JF, et al. Glial growth factor 2 promotes functional recovery with treatment initiated up to 7 days after permanent focal ischemic stroke. Neuropharmacology. 2010;59(7-8):640-9.

5. Guo WP, et al. Neuroprotective effects of neuregulin-1 in rat models of focal cerebral ischemia. Brain Res. 2006;1087(1):180-5.

6. Shyu WC, et al. Neuregulin-1 reduces ischemia-induced brain damage in rats. Neurobiol Aging. 2004;25(7):935-44.

7. $\mathrm{Xu} \mathrm{Z}$, et al. Neuregulin-1 is neuroprotective and attenuates inflammatory responses induced by ischemic stroke. Biochem Biophys Res Commun. 2004:322(2):440-6.

8. Li Q, et al. Effect of neuregulin on apoptosis and expressions of STAT3 and GFAP in rats following cerebral ischemic reperfusion. J Mol Neurosci. 2009; 37(1):67-73.

9. Solomon W, et al. Neuregulin-1 attenuates mortality associated with experimental cerebral malaria. J Neuroinflammation. 2014;11:9.

10. Li Y, et al. Neuregulin-1 inhibits neuroinflammatory responses in a rat model of organophosphate-nerve agent-induced delayed neuronal injury. J Neuroinflammation. 2015;12:64.

11. Dimayuga FO, et al. The neuregulin GGF2 attenuates free radical release from activated microglial cells. J Neuroimmunol. 2003;136(1-2):67-74.

12. Touzani O, Roussel S, Mackenzie ET. The ischaemic penumbra. Curr Opin Neurol. 2001;14(1):83-8.

13. Dirnagl U, ladecola C, Moskowitz MA. Pathobiology of ischaemic stroke: an integrated view. Trends Neurosci. 1999;22(9):391-7.

14. Rogove AD, Lu W, Tsirka SE. Microglial activation and recruitment, but not proliferation, suffice to mediate neurodegeneration. Cell Death Differ. 2002; 9(8):801-6.

15. Graeber MB, Streit WJ. Microglia: biology and pathology. Acta Neuropathol. 2010;119(1):89-105.

16. McKimmie CS, et al. Innate immune response gene expression profiles of N9 microglia are pathogen-type specific. J Neuroimmunol. 2006;175(1-2):128-41.

17. Jin R, Yang G, Li G. Inflammatory mechanisms in ischemic stroke: role of inflammatory cells. J Leukoc Biol. 2010;87(5):779-89.

18. Fernandes A, Miller-Fleming L, Pais TF. Microglia and inflammation: conspiracy, controversy or control? Cell Mol Life Sci. 2014;71(20):3969-85.

19. Graeber MB, Li W, Rodriguez ML. Role of microglia in CNS inflammation. FEBS Lett. 2011;585(23):3798-805.

20. Nathan C, Ding A. Nonresolving inflammation. Cell. 2010;140(6):871-82.

21. Widgerow AD. Cellular resolution of inflammation-catabasis. Wound Repair Regen. 2012;20(1):2-7.

22. Nurmi A, et al. Nuclear factor-kappaB contributes to infarction after permanent focal ischemia. Stroke. 2004;35(4):987-91.

23. Hayden MS, Ghosh S. Shared principles in NF-kappaB signaling. Cell. 2008; 132(3):344-62.

24. Ridder DA, Schwaninger M. NF-kappaB signaling in cerebral ischemia. Neuroscience. 2009;158(3):995-1006.

25. Chen CC, et al. Protein kinase $C$ eta mediates lipopolysaccharide-induced nitric-oxide synthase expression in primary astrocytes. J Biol Chem. 1998; 273(31):19424-30

26. Boulet I, et al. Lipopolysaccharide- and interferon-gamma-induced expression of hck and lyn tyrosine kinases in murine bone marrow-derived macrophages. Oncogene. 1992;7(4):703-10.

27. Ko HM, et al. Inflexin attenuates proinflammatory responses and nuclear factor-kappaB activation in LPS-treated microglia. Eur J Pharmacol. 2010; 633(1-3):98-106

28. Jin $\mathrm{H}$, et al. Myrislignan attenuates lipopolysaccharide-induced inflammation reaction in murine macrophage cells through inhibition of NF-kappaB signalling pathway activation. Phytother Res. 2012;26(9):1320-6.

29. Wang X, et al. Gomisin A inhibits lipopolysaccharide-induced inflammatory responses in N9 microglia via blocking the NF-kappaB/MAPKs pathway. Food Chem Toxicol. 2014;63:119-27.

30. Sun SC. Non-canonical NF-kappaB signaling pathway. Cell Res. 2011;21(1):71-85. 
31. Lawrence T, et al. IKKalpha limits macrophage NF-kappaB activation and contributes to the resolution of inflammation. Nature. 2005;434(7037):1138-43.

32. Bonizzi G, Karin M. The two NF-kappaB activation pathways and their role in innate and adaptive immunity. Trends Immunol. 2004;25(6):280-8.

33. Xiao G, et al. Alternative pathways of NF-kappaB activation: a double-edged sword in health and disease. Cytokine Growth Factor Rev. 2006;17(4):281-93.

34. Xiao G, Harhaj EW, Sun SC. NF-kappaB-inducing kinase regulates the processing of NF-kappaB2 p100. Mol Cell. 2001;7(2):401-9.

35. Lawrence $\mathrm{T}$. The nuclear factor NF-kappaB pathway in inflammation. Cold Spring Harb Perspect Biol. 2009;1(6):a001651.

36. Marienfeld $\mathrm{R}$, et al. RelB forms transcriptionally inactive complexes with RelA/p65. J Biol Chem. 2003;278(22):19852-60.

37. Lwin $\mathrm{T}$, et al. Bone marrow stromal cells prevent apoptosis of lymphoma cells by upregulation of anti-apoptotic proteins associated with activation of NF-kappaB (RelB/p52) in non-Hodgkin's lymphoma cells. Leukemia. 2007; 21(7):1521-31.

38. Zong WX, et al. The prosurvival Bcl-2 homolog Bfl-1/A1 is a direct transcriptional target of NF-kappaB that blocks TNFalpha-induced apoptosis. Genes Dev. 1999;13(4):382-7.

39. Xia $Y$, et al. RelB regulation of chemokine expression modulates local inflammation. Am J Pathol. 1997;151(2):375-87.

40. Limpert AS, Carter BD. Axonal neuregulin 1 type III activates NF-kappaB in Schwann cells during myelin formation. J Biol Chem. 2010;285(22):16614-22.

41. Hinohara $\mathrm{K}$, et al. ErbB receptor tyrosine kinase/NF-kappaB signaling controls mammosphere formation in human breast cancer. Proc Natl Acad Sci U S A 2012;109(17):6584-9.

42. Limpert AS, et al. NF-kappaB forms a complex with the chromatin remodeler BRG1 to regulate Schwann cell differentiation. J Neurosci. 2013;33(6):2388-97.

43. Frensing T, Kaltschmidt C, Schmitt-John T. Characterization of a neuregulin1 gene promoter: positive regulation of type I isoforms by NF-kappaB. Biochim Biophys Acta. 2008;1779(2):139-44.

44. Han ME, et al. Overexpression of NRG1 promotes progression of gastric cancer by regulating the self-renewal of cancer stem cells. J Gastroenterol. 2015;50(6):645-56

45. Bhat-Nakshatri P, Sweeney CJ, Nakshatri H. Identification of signal transduction pathways involved in constitutive NF-kappaB activation in breast cancer cells. Oncogene. 2002;21(13):2066-78.

46. Chen $\mathrm{D}$, et al. NIK is a component of the EGF/heregulin receptor signaling complexes. Oncogene. 2003;22(28):4348-55.

47. Karanam S, Moreno CS. CONFAC: automated application of comparative genomic promoter analysis to DNA microarray datasets. Nucleic Acids Res. 2004;32(Web Server issue):W475-84.

48. Pulliam JV, et al. Computational identification of conserved transcription factor binding sites upstream of genes induced in rat brain by transient focal ischemic stroke. Brain Res. 2013;1495:76-85.

49. Ford G, et al. Expression Analysis Systematic Explorer (EASE) analysis reveals differential gene expression in permanent and transient focal stroke rat models. Brain Res. 2006;1071(1):226-36.

50. Harari OA, Liao JK. NF-kappaB and innate immunity in ischemic stroke. Ann N Y Acad Sci. 2010;1207:32-40.

51. Acosta SA, et al. Combination therapy of human umbilical cord blood cells and granulocyte colony stimulating factor reduces histopathological and motor impairments in an experimental model of chronic traumatic brain injury. PLoS One. 2014;9(3):e90953.

52. Shin YK, Cho SR. Exploring erythropoietin and G-CSF combination therapy in chronic stroke patients. Int J Mol Sci. 2016;17:4.

53. Sikoglu EM, et al. Enhancement in cognitive function recovery by granulocyte-colony stimulating factor in a rodent model of traumatic brain injury. Behav Brain Res. 2014;259:354-6.

54. Chen C, Edelstein LC, Gelinas C. The Rel/NF-kappaB family directly activates expression of the apoptosis inhibitor Bcl-x(L). Mol Cell Biol. 2000;20(8):2687-95.

55. Yoza BK, et al. Induction of RelB participates in endotoxin tolerance. J Immunol. 2006;177(6):4080-5.

56. Hayden MS, Ghosh S. Signaling to NF-kappaB. Genes Dev. 2004;18(18):2195-224.

57. Guan YF, et al. Neuregulin 1 protects against ischemic brain injury via ErbB4 receptors by increasing GABAergic transmission. Neuroscience. 2015;307:151-9.

58. Fontaine $\mathrm{RH}$, et al. IL-9/IL-9 receptor signaling selectively protects cortical neurons against developmental apoptosis. Cell Death Differ. 2008;15(10): 1542-52.
59. Guo Y, et al. Granulocyte colony-stimulating factor improves alternative activation of microglia under microenvironment of spinal cord injury. Neuroscience. 2013;238:1-10.

60. Yamasaki $\mathrm{R}$, et al. Restoration of microglial function by granulocyte-colony stimulating factor in ALS model mice. J Neuroimmunol. 2010;229(1-2):51-62.

61. Richard $M$, et al. Interleukin-9 regulates NF-kappaB activity through BCL3 gene induction. Blood. 1999;93(12):4318-27.

62. Bundy DL, McKeithan TW. Diverse effects of BCL3 phosphorylation on its modulation of NF-kappaB p52 homodimer binding to DNA. J Biol Chem. 1997;272(52):33132-9.

63. Gao R, et al. A phase II, randomized, double-blind, multicenter, based on standard therapy, placebo-controlled study of the efficacy and safety of recombinant human neuregulin-1 in patients with chronic heart failure. J Am Coll Cardiol. 2010;55(18):1907-14.

64. Jabbour $A$, et al. Parenteral administration of recombinant human neuregulin-1 to patients with stable chronic heart failure produces favourable acute and chronic haemodynamic responses. Eur J Heart Fail. 2011;13(1):83-92.

\section{Submit your next manuscript to BioMed Central and we will help you at every step:}

- We accept pre-submission inquiries

- Our selector tool helps you to find the most relevant journal

- We provide round the clock customer support

- Convenient online submission

- Thorough peer review

- Inclusion in PubMed and all major indexing services

- Maximum visibility for your research

Submit your manuscript at www.biomedcentral.com/submit 